\title{
Symptomatic CNS Radiation Necrosis Requiring Neurosurgical Resection During Treatment with Lorlatinib in ALK-Rearranged NSCLC: A Report of Two Cases
}

This article was published in the following Dove Press journal:

Lung Cancer: Targets and Therapy

Viola W Zhu (D) ${ }^{1, *}$

Misako Nagasaka (iD) $2,3, *$

Takafumi Kubota (iD) 4

Kunil Raval ${ }^{5}$

Natasha Robinette (iD ${ }^{6}$

Octavio Armas ${ }^{7}$

Wajd Al-Holou ${ }^{8}$

Sai-Hong Ignatius Ou'

'Chao Family Comprehensive Cancer Center, Division of Hematology/Oncology, Department of Medicine, University of California Irvine, Orange, CA, USA;

${ }^{2}$ Department of Oncology, Karmanos

Cancer Institute/Wayne State University,

Detroit, MI, USA; ${ }^{3}$ Department of

Advanced Medical Innovations, St.

Marianna University Graduate School of

Medicine, Kawasaki, Japan; ${ }^{4}$ Department of

Neurology, Sapporo Nishimaruyama

Hospital, Sapporo, Japan; ${ }^{5}$ Department of

Pathology, Wayne State University, Detroit, MI, USA; ${ }^{6}$ Department of Oncology, Imaging Division, Karmanos Cancer Institute, Wayne State University, Detroit, MI, USA; ' ${ }^{D}$ epartment of Pathology, Sharp Grossmont Hospital, La Mesa, CA, USA;

${ }^{8}$ Department of Neurosurgery, Karmanos Cancer Institute/Wayne State University,

Detroit, MI, USA

*These authors contributed equally to this work

Correspondence: Sai-Hong lgnatius Ou Chao Family Comprehensive Cancer

Center, Department of Medicine, Division of Hematology/Oncology, University of

California Irvine, 200 Manchester Dr, Suite

410 , Orange, CA 92868, USA

$\mathrm{Tel}+|7| 4-456-8104$

Fax +|7|4-456-2242

Email siou@uci.edu

\begin{abstract}
Central nervous system (CNS) metastasis carries a significant morbidity and mortality in anaplastic lymphoma kinase $(A L K)$-rearranged non-small cell lung cancer (NSCLC). Next-generation ALK tyrosine kinase inhibitors (TKIs) are highly CNS-penetrant and have demonstrated remarkable intracranial activity across clinical studies, and yet radiation remains the mainstay of treatment modality against CNS metastasis. We have previously reported alectinib can induce CNS radiation necrosis even after a remote history of radiation (7 years post-radiation). Lorlatinib is another potent next-generation ALK TKI that can overcome many $A L K$ resistance mutations and has been shown to have excellent activity in patients with baseline CNS metastasis. Here we report two $A L K$-rearranged NSCLC patients who developed radiation necrosis shortly after initiating lorlatinib following progression on the sequential treatment of crizotinib, alectinib, and brigatinib. In both cases, radiation necrosis is evidenced by serial MRI images and histological examination of the resected CNS metastasis that had previously been radiated. Our cases highlight the importance of recognizing CNS radiation necrosis that may mimic disease progression in $A L K$-rearranged NSCLC treated with and potentially precipated by next-generation ALK TKIs.
\end{abstract}

Keywords: CNS, radiation necrosis, lorlatinib, ALK-rearranged NSCLC, alectinib

\section{Introduction}

Central nervous system (CNS) metastasis carries a significant morbidity and mortality in anaplastic lymphoma kinase $(A L K)$-rearranged non-small cell lung cancer (NSCLC). Among the three most common receptor tyrosine kinase (RTK) fusions in NSCLC ( $A L K, R O S 1, R E T), A L K$-rearranged NSCLC has the highest cumulative incidence of CNS metastasis over the course of the disease process with a projected cumulative incidence of $65 \%$ at 6 years from diagnosis. ${ }^{1}$ Lorlatinib is a highly CNS-penetrant and potent third-generation ALK tyrosine kinase inhibitor (TKI) that can overcome almost all of the crizotinib-resistant $A L K$ mutations including C1156Y, I1171N/S/T, F1174C, L1196M, D1203N, G1269A, as well as the solvent front mutation G1202R. ${ }^{2,3}$ Given its excellent CNS penetration, the efficacy of lorlatinib has been observed even in patients with CNS metastasis after disease progression on other second-generation ALK TKIs. ${ }^{4,5}$ Lorlatinib has been approved by the US FDA in November 2018 for the treatment of advanced $A L K$-rearranged NSCLC after progression on first-line alectinib or ceritinib or after crizotinib and at 
least one other ALK TKI. ${ }^{6}$ While oligometastatic CNS metastasis could be considered for surgical resection when anatomically feasible, lung cancer patients are oftentimes found with multiple CNS metastases that are typically treated with radiation (whole brain or stereotactic radiation). Thus, lorlatinib is frequently used in $A L K$ rearranged NSCLC patients with previously radiated CNS metastasis. Here we report two cases of radiation necrosis requiring neurosurgical resection during lorlatinib treatment following disease progression on secondgeneration ALK TKIs.

\section{Case Presentation}

Case 1 patient is a 45 -year-old Caucasian woman with a former light-smoking history who presented with stage IV lung adenocarcinoma including CNS metastasis, bilateral lung involvement, and left iliac bony metastasis. Stereotactic radiosurgery (SRS) was given to the right frontal lobe, right occipital lobe, and right cerebellar lesions (2000 cGy in a single fraction to each lesion; Figure 1A). Molecular profiling of the lung mass was positive for $A L K$ by FISH (62\% split signal) and negative for activating EGFR mutations and ROS1 fusion. Crizotinib at $250 \mathrm{mg}$ twice daily was started but it was switched to alectinib 9 months later due to CNS progression. However, after being on alectinib for 6 months, CNS progression continued with worsening known brain lesions (Figure 1A) and a new left frontal lobe lesion for which whole-brain radiation (WBRT) was given (3750 cGy in fifteen fractions). Alectinib was held during WBRT and then switched to brigatinib which achieved stable CNS control for 4 months until a new left cerebellar lesion appeared. This lesion subsequently resolved 2 months after switching to lorlatinib, but 3 additional months later the patient presented with headaches, nausea, and vomiting and was found to have worsening edema around the right frontal lobe lesion (Figure 1A). She underwent neurosurgical resection of this lesion and pathology was consistent with radiation necrosis (Figure 1B). Lorlatinib was held for 1 week peri-operatively. Six months after resuming lorlatinib, the patient again developed headaches due to worsening edema around the right occipital lobe lesion (Figure 1A). Pathology from the second resection was again consistent with radiation necrosis (Figure 1B). The patient has remained on lorlatinib since. Throughout the treatment course, her extracranial disease was responding to various ALK TKIs with a confirmed partial response (PR).

Case 2 patient is a 75-year-old never-smoking African American woman who was diagnosed with stage IV lung adenocarcinoma in October 2006. She had received multiple lines of chemotherapy until July 2014 when a biopsy of a liver lesion revealed an $E M L 4-A L K$ variant 1 fusion. She achieved a PR on crizotinib for 16 months. Due to CNS progression, crizotinib was switched to alectinib and she achieved a PR for another 27 months until further progression of a left frontal lobe lesion (Figure 2A). SRS was given to this lesion (1800 cGy in single fraction) and three other small lesions while continuing alectinib. Three months later, she received additional SRS (2000 cGy in single fraction each) to six new asymptomatic small CNS lesions. Subsequently, alectinib was switched to brigatinib but the first surveillance scan after 7 weeks of treatment with brigatinib revealed an increase in the size of the left frontal lobe lesion with increased surrounding edema (Figure 2A), along with three additional punctate enhancing lesions. Within 2 weeks of switching from brigatinib to lorlatinib, the patient developed altered mental status due to further increased edema around the left frontal lobe lesion (Figure 2A). Pathology from resection of this lesion revealed radiation necrosis (Figure 2B). Currently, she is on a reduced dose of lorlatinib at $50 \mathrm{mg}$ once daily. The schematic summary of the treatment course of each case is shown in Figures $1 \mathrm{C}$ and $2 \mathrm{C}$ respectively. Both patients have provided a written informed consent to have the case details published and institutional approval was not required.

\section{Discussion}

Case 1 illustrates a relatively common clinical scenario with RTK fusion-driven lung cancer where there may be continuous intracranial progression despite extracranial disease response with various ALK TKIs. Repeated radiation is often delivered to treat new and/or progressing CNS metastasis. In the randomized ALEX trial comparing the first-line crizotinib with alectinib, the cumulative incidence of $\mathrm{CNS}$ metastasis at 12 months was $8.6 \%$ for alectinib and $50.4 \%$ for crizotinib among patients who had received prior radiation for CNS metastasis. ${ }^{7}$ Lorlatinib has demonstrated significant intracranial activity even after disease progression on alectinib or ceritinib with an intracranial response rate of $63.0 \%$ and a median duration of intracranial response of 14.5 months in $A L K$-rearranged NSCLC with exposure to at least one prior ALK TKI. ${ }^{5}$ We have previously reported that radiation necrosis necessitating neurosurgical resection could occur with alectinib. ${ }^{8,9}$ In one case, radiation necrosis occurred after 7 years from the last radiation treatment and 12 months into alectinib treatment. ${ }^{9}$ While radiation necrosis did not occur during alectinib treatment in our case 1 
A

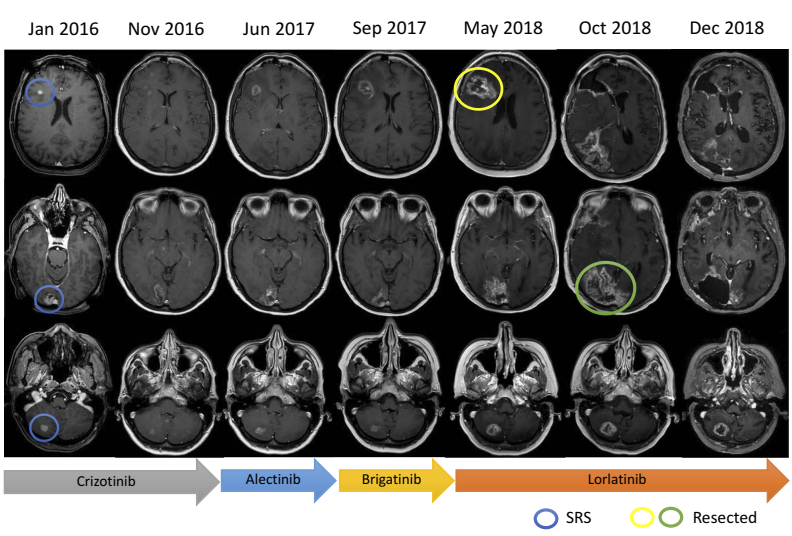

B

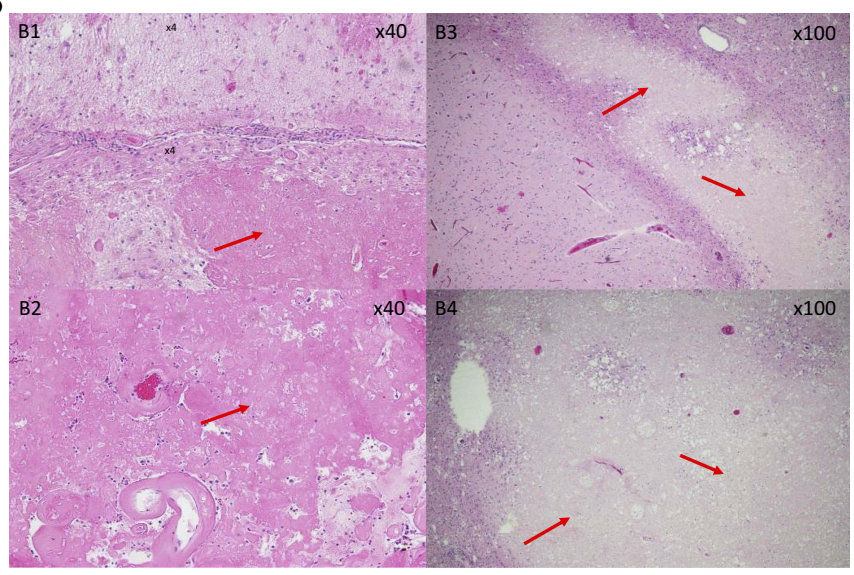

\section{C}

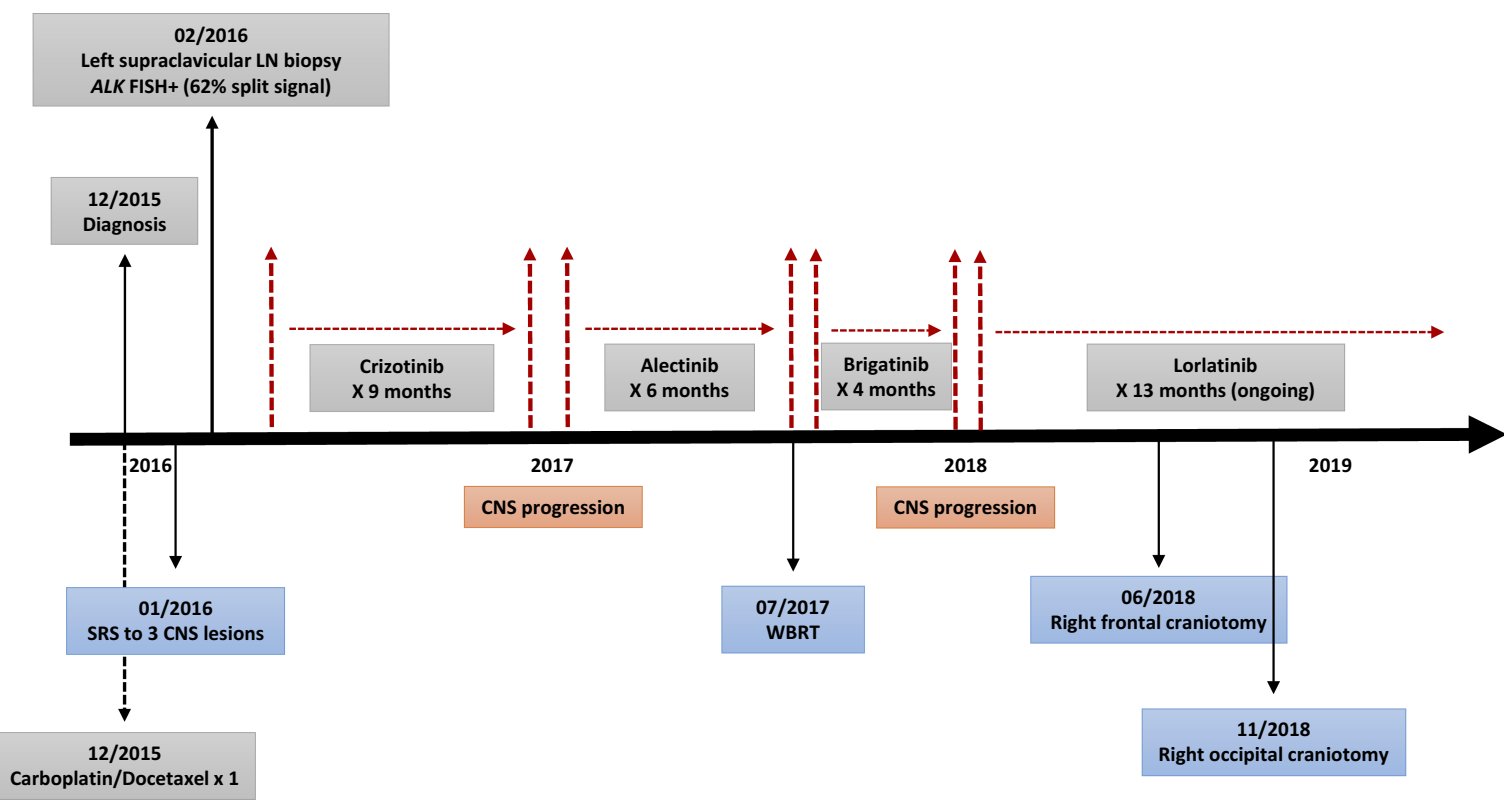

Figure I (A) Serial MRI of the brain demonstrating intracranial lesions treated with stereotactic radiosurgery (SRS) (blue circles) and craniotomy (yellow and green circles). Progressive cerebral edema in the right frontal and occipital lobes during lorlatinib treatment is shown. Jan 2016: prior to SRS; Nov 2016: progression on crizotinib; Jun 20I7: progression on alectinib prior to whole-brain radiation; Sep 2017: progression on brigatinib; May 2018: radiation necrosis \#I; Oct 2018: radiation necrosis \#2; Dec 20I 8: lorlatinib resumed post-operatively. (B) Histological slides (40X magnification) from the first craniotomy revealed extensive necrosis of gray matter (BI) and severe hyalinization of white matter with surrounding necrosis (B2). Histological slides (I00X magnification) from the second craniotomy also demonstrated gray matter sparing and white matter necrosis (B3). Note the vessels in the background of extensive white matter necrosis (B4). Red arrows point towards areas of necrosis. (C) Schematic summary of the treatment course. Abbreviations: LN, lymph node; SRS, stereotactic radiosurgery; WBRT, whole-brain radiation.

patient, symptomatic radiation necrosis occurred twice both requiring neurosurgical resection during lorlatinib treatment with the second episode of radiation necrosis occurring after 11 months into lorlatinib treatment. Radiation necrosis is a known side effect of radiation treatment. ${ }^{10,11}$ While in case 1 both radiation necrosis could have been attributed to radiation itself, we hypothesize that lorlatinib may have conceivably accelerated the development of radiation necrosis. Due to its potent ALK inhibitory activity, any residual tumor growth in previously radiated CNS metastasis could have been inhibited by lorlatinib potentially even triggering further tumor necrosis. In case 1 , we could not rule out the possibility of radiation necrosis starting at the time of switching from alectinib to brigatinib, but the appearance of three new punctate lesions necessitated the transition from brigatinib to lorlatinib due to limited clinical efficacy data on sequencing from alectinib to brigatinib. ${ }^{12}$ Radiation necrosis rapidly developed 2 weeks after initiating lorlatinib. As such, there remains a possibility of lorlatinib accelerating the occurrence of radiation necrosis potentially initiated during brigatinib treatment.

Concurrent use of EGFR TKIs with upfront WBRT to SRS has been identified as a significant risk factor for developing radiation necrosis. ${ }^{13}$ Interestingly, $A L K$-rearranged 
A

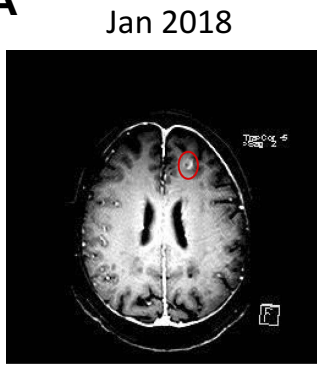

Apr 2018

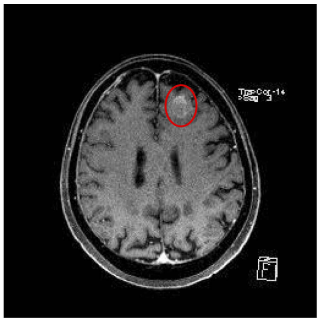

Jul 2018

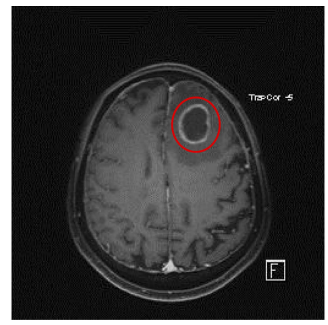

Oct 2018

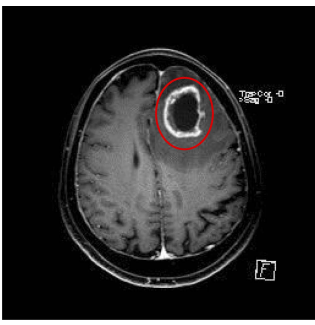

Jan 2019

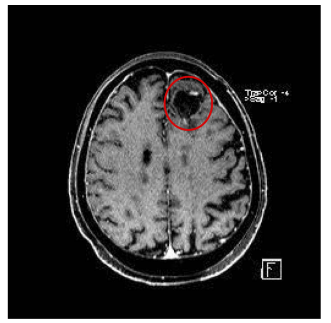

\section{Alectinib}
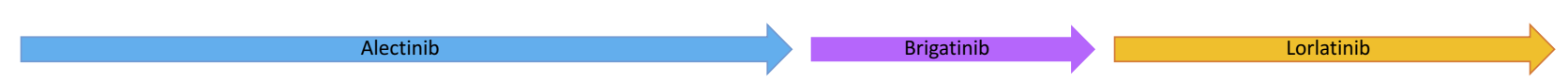

B
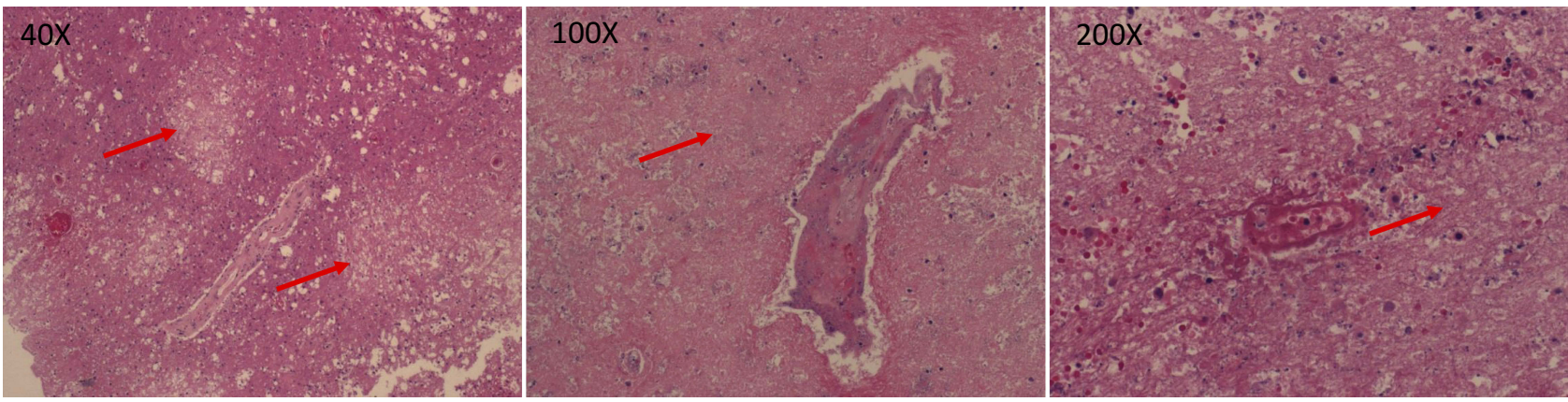

C
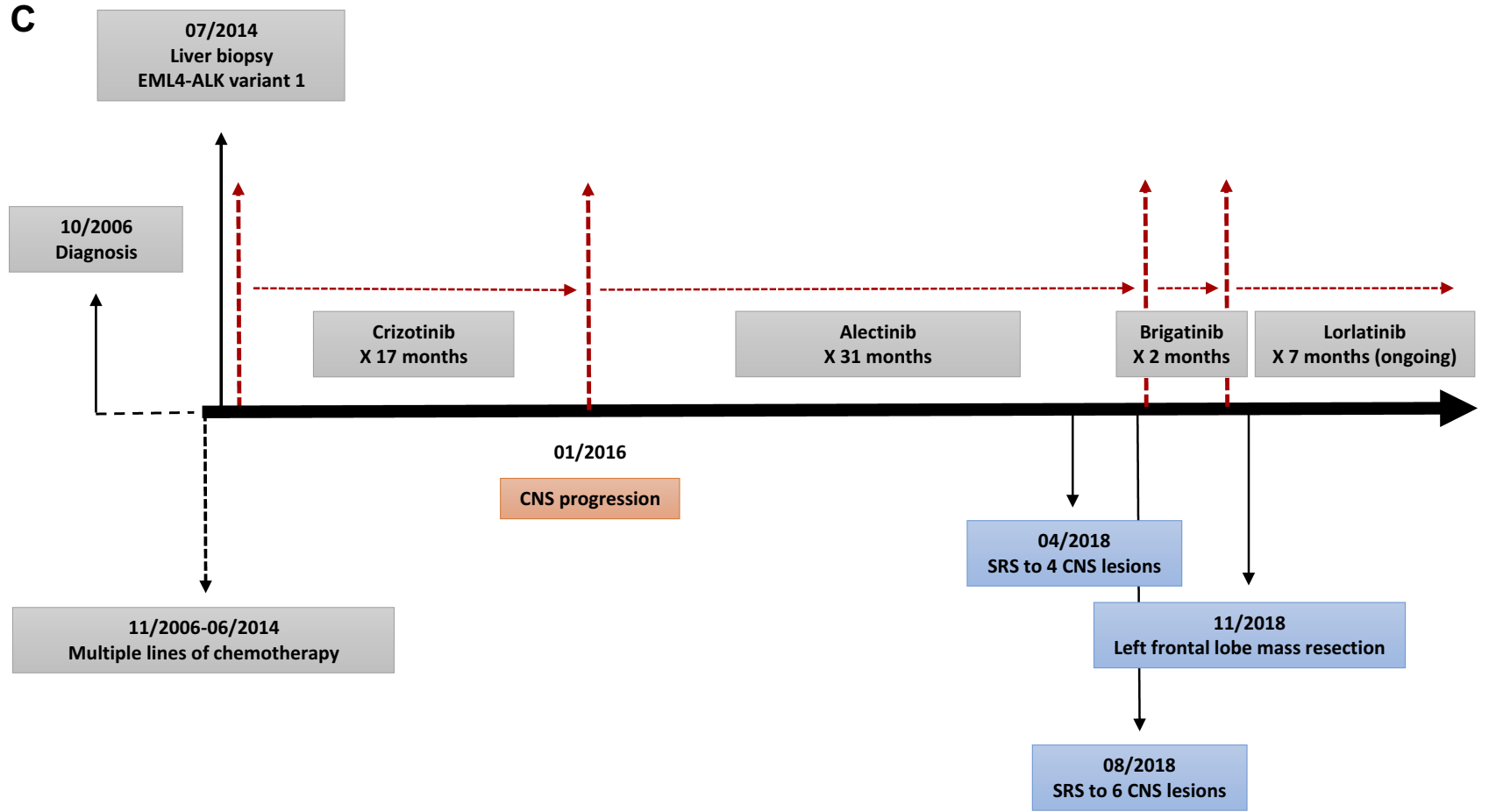

$11 / 2006-06 / 2014$

Multiple lines of chemotherapy

$01 / 2016$

Figure 2 (A) MRI of the brain demonstrating the left frontal lobe lesion indicated by the red circles over the course of sequential treatment with alectinib, brigatinib, and lorlatinib. Jan 2018: the enhancing left superior frontal lesion while on alectinib; Apr 2018: progression of the left superior frontal lesion leading to stereotactic radiosurgery (SRS) while on alectinib; Jul 2018: alectinib switched to brigatinib due to asymptomatic CNS progression (not shown on this slice of scan) leading to SRS; Oct 20I8: brigatinib switched to lorlatinib followed by radiation necrosis; Jan 20I9: lorlatinib resumed post-operatively. (B) Histological slides (40X, I00X, 200X magnification) from the resected CNS lesion showed extensive necrosis of gray matter. Red arrows point towards areas of necrosis. (C) Schematic summary of the treatment course.

Abbreviation: SRS, stereotactic radiosurgery. 
NSCLC has also been identified as a risk factor for developing radiation necrosis (hazard ratio of 6.36). ${ }^{14}$ Large-scale multi-institutional analyses will be required to determine whether the increased risk of radiation necrosis in $A L K$ rearranged NSCLC is the result of inherent tumor biology, a high incidence of CNS metastasis, repeated CNS radiation, systemic treatment with highly CNS-penetrant ALK TKIs, or a combination of these factors. When feasible, upfront use of CNS-penetrant ALK TKIs prior to radiation even in patients with untreated large or symptomatic CNS metastasis should be explored. ${ }^{15}$

\section{Conclusion}

With the advent of multiple ALK TKIs, the median overall survival of $A L K$-rearranged NSCLC patients treated with sequential ALK TKIs can be up to 7.5 years; ${ }^{16}$ and the median overall survival from stage IV disease can be at least 6.8 years. ${ }^{17} A L K$-rearranged NSCLC patients are likely to develop CNS metastasis during their disease course requiring radiation treatment. Acknowledging the fact that radiation necrosis could occur during a later line of lorlatinib treatment is critical as it often mimics disease progression which may lead to premature discontinuation of lorlatinib.

\section{Disclosure}

VWZ has received honoraria from AstraZeneca, Biocept, Roche-Foundation Medicine, Roche/Genentech, Takeda, and consulting fees from TP Therapeutics, outside the submitted work. MN has received honoraria from AstraZeneca and Tempus, outside the submitted work. SIO has previously served on the Scientific Advisory Board of Turning Point Therapeutics with stock ownership; has received honoraria from AstraZeneca, Pfizer, Merck, Novartis, RocheFoundation Medicine, Roche/Genentech, Spectrum, Takeda, outside the submitted work. The authors report no other conflicts of interest in this work.

\section{References}

1. Drilon A, Lin JJ, Filleron T, et al. Frequency of brain metastases and multikinase inhibitor outcomes in patients with RET-rearranged lung cancers. J Thorac Oncol. 2018;13:1595-1601. doi:10.1016/j. jtho.2018.07.004

2. Gainor JF, Dardaei L, Yoda S, et al. Molecular mechanisms of resistance to first- and second-generation ALK inhibitors in ALK-rearranged lung cancer. Cancer Discov. 2016;6:1118-1133. doi:10.1158/2159-8290.CD-16-0596
3. Basit S, Ashraf Z, Lee K, et al. First macrocyclic 3rd-generation ALK inhibitor for treatment of ALK/ROD1 cancer: clinical and designing strategy update of lorlatinib. Eur $J$ Med Chem. 2017;134:348-356. doi:10.1016/j.ejmech.2017.04.032

4. Zou HY, Friboulet L, Kodack DP, et al. PF-06463922, an ALK/ROS1 inhibitor, overcomes resistance to first and second generation ALK inhibitors in preclinical models. Cancer Cell. 2015;28:70-81. doi:10.1016/j.ccell.2015.05.010

5. Solomon BJ, Besse B, Bauer TM, et al. Lorlatinib in patients with $A L K$-positive non-small-cell lung cancer: results from a global Phase 2 study. Lancet Oncol. 2018;19:1654-1667. doi:10.1016/S14702045(18)30649-1

6. Syed YY. Lorlatinib: first global approval. Drugs. 2019;79:93-98. doi:10.1007/s40265-018-1041-0

7. Gadgeel S, Peters S, Mok T, et al. Alectinib versus crizotinib in treatment-naïve anaplastic lymphoma kinase-positive $(A L K+)$ nonsmall-cell lung cancer: CNS efficacy results from the ALEX study. Ann Oncol. 2018;29:2214-2222. doi:10.1093/annonc/mdy405

8. Ou SH, Klempner SJ, Azada MC, et al. Radiation necrosis presenting as pseudoprogression (PsP) during alectinib treatment of previously radiated brain metastases in $A L K$-positive NSCLC: implications for disease assessment and management. Lung Cancer. 2015;88:355-359. doi:10.1016/j.lungcan.2015.03.022

9. Ou SH, Weitz M, Jalas JR, et al. Alectinib induced CNS radiation necrosis in an $A L K+$ NSCLC patient with a remote (7 years) history of brain radiation. Lung Cancer. 2016;96:15-18. doi:10.1016/j. lungcan.2016.03.008

10. Vellayappan B, Tan CL, Yong C, et al. Diagnosis and management of radiation necrosis in patients with brain metastases. Front Oncol. 2018;8:395. doi:10.3389/fonc.2018.00395

11. Sneed PK, Mendez J, Vemer-van den Hoek JM, et al. Adverse radiation effect after stereotactic radiosurgery for brain metastases: incidence, time course, and risk factors. $J$ Neurosurg. 2015;123:373-386. doi:10.3171/2014.10.JNS141610

12. Lin JJ, Zhu VW, Schoenfeld AJ, et al. Brigatinib in patients with alectinib-refractory $A L K$-positive NSCLC. J Thorac Oncol. 2018;13:1530-1538. doi:10.1016/j.jtho.2018.06.005

13. Kim JM, Miller JA, Kotecha R, et al. The risk of radiation necrosis following stereotactic radiosurgery with concurrent systemic therapies. J Neurooncol. 2017;133:357-368. doi:10.1007/s11060017-2442-8

14. Miller JA, Bennett EE, Xiao R, et al. Association between radiation necrosis and tumor biology after stereotactic radiosurgery for brain metastasis. Int J Radiat Oncol Biol Phys. 2016;96:1060-1069. doi:10.1016/j.ijrobp.2016.08.039

15. Lin JJ, Jiang GY, Joshipura N, et al. Efficacy of alectinib in patients with $A L K$-positive NSCLC and symptomatic or large CNS metastases. J Thorac Oncol. 2019;14:683-690. doi:10.1016/j. jtho.2018.12.002

16. Duruisseaux M, Besse B, Cadranel J, et al. Overall survival with crizotinib and next-generation ALK inhibitors in $A L K$-positive non-small-cell lung cancer (IFCT-1302 CLINALK): a French nationwide cohort retrospective study. Oncotarget. 2017;83:21903-21917.

17. Pacheco JM, Gao D, Smith D, et al. Natural history and factors associated with overall survival in stage IV $A L K$-rearranged non-small cell lung cancer. $J$ Thorac Oncol. 2019;14:691-700. doi:10.1016/j.jtho.2018.12.014 


\section{Publish your work in this journal}

Lung Cancer: Targets and Therapy is an international, peerreviewed, open access journal focusing on lung cancer research, identification of therapeutic targets and the optimal use of preventative and integrated treatment interventions to achieve improved outcomes, enhanced survival and quality of life for the cancer patient. Specific topics covered in the journal include: Epidemiology,

Submit your manuscript here: http://www.dovepress.com/lung-cancer-targets-therapy-journal detection and screening; Cellular research and biomarkers; Identification of biotargets and agents with novel mechanisms of action; Optimal clinical use of existing anticancer agents, including combination therapies; Radiation and surgery; Palliative care; Patient adherence, quality of life, satisfaction; Health economic evaluations. 